\title{
Thermal and Dynamical Structures of Convective Motions in a Rotating Fluid Annulus Subject to Internal Heating
}

\author{
By Kazuo Ukaji \\ Department of Applied Physics, Fukuoka University, Fukuoka, Japan \\ (Manuscript received 30 July 1979, in revised form 5 November 1979)
}

\begin{abstract}
Thermal and dynamical structures of the convective motions in a rotating fluid annulus, heated internally by passing an a.c. current through the fluid, are studied experimentally.

The effects of internal heating are seen in the following results.

1) The measurements of the rate of radial heat transport to the inner wall and the drift angular velocity of the vortex disturbances show that they depend on the rotation rate $\Omega$ in a similar way, with the minimum at a certain rotation rate $\Omega_{m}$.

2) The distributions of the zonal mean temperature show that, when $\Omega>\Omega_{m}$, the region nearby the mean radius of the fluid layer becomes warmer than the inner and outer regions at the same height.

3) The mean zonal flow of the wave motion for $\Omega>\Omega_{m}$, as well as the axisymmetric zonal flows, has the distribution of the vertical component of vorticity with negative values over a wide region at the top-surface.

4) The amplitudes of the temperature and pressure deviations increase with $\Omega$ and, near $\Omega_{m}$, the inclination of the axes of the temperature deviation from the vertical direction becomes least and the most active outward heat transport due to the vortex disturbances takes place.

The analysis shows that the fundamental structures of the vortex disturbances developing in the internally heated system are the same as those of the baroclinic waves analyzed by Matsuwo et al. $(1976,1977)$ in the wall-heated system. Therefore, the indication by Hide and Mason (1970), namely, that the principal characteristics of the flow (in the rotating fluid annulus) are fairly insensitive to the radial dependence of heating and cooling, is supported from the view-point of structure analysis.
\end{abstract}

\section{Introduction}

Laboratory experiments of thermal convection in a rotating fluid annulus with a radial temperature gradient have been subjects of several researchers. Hide (1958) showed the existence of four different types of flows: axisymmetric zonal flow, steady waves, vacillating flow and irregular flow. The regime diagram of these flows was established by Fowlis and Hide (1965) and, for each flow, measurements of radial heat transport, temperature fields and velocity fields have been carried out (Bowden and Eden, 1965; Kaiser, 1969, 1971; Ketchem, 1972; Uryu et al., 1974). Recently, Matsuwo et al. (1976, 1977) analyzed the internal structure of the wave motion in detail by the use of a large annulus.
In these experiments, a radial temperature gradient is set up by heating one side-wall and cooling another side-wall of the annular convection chamber. The realized temperature distribution in the rotating fluid layer is almost linear in the radial direction in such wall-heated systems (Bowden and Eden, 1965; Matsuwo et al., 1976).

On the other hand, Hide and Mason (1970) intended to set up a parabolic temperature distribution by adopting an internally heated system, which consists of generation of heat by passing an a.c. current through the fluid and removal of heat via one side-wall or both side-walls simultaneously. They obtained results similar to those found in wall-heating experiments, namely, that the four distinct types of flow stated above are 
possible. The "upper transition" occurs in agreement with Eady's theory (1949). The Nusselt number decreases with the increase of the basic rotation rate in the axisymmetric regime, but increases a little when wave motions develop. In addition to these results, they also indicated that the top-surface flow patterns in the internally heated system have characteristic features. In the case with both walls cooled, the azimuthal velocity in the axisymmetric regime reverses sigh on a certain circumference between the inner and outer walls and the anticlockwise vortices without the "jet" stream develop in the steady wave regime.

Their experiments consist of flow observations and temperature measurements. But, the temperature measurements were limited to those at several fixed points so that the structure analysis of the flows has been left to be studied. In this paper, we analyze the three-dimensional structure of thermal convection in a rotating fluid annulus generated by internal heating and heat removal through both side-walls. The general characteristics, which furnished a guide to the structure analysis, are also presented.

Although this work was motivated by a hydrodynamical interest, we expect that the results obtained play a role to understand some kinds of atmospheric disturbances, e.g., the monsoon disturbances in low latitudes.

\section{Experimental apparatus}

The main parts of the experimental apparatus are schematically shown in Fig. 1. Three concentric cylinders $\mathbf{A}, \mathbf{B}$ and $\mathbf{C}$, each $0.2 \mathrm{~cm}$ thick and $19.5 \mathrm{~cm}$ high, are mounted on a polyvinyl chloride disk $\mathbf{D}$ of $2.0 \mathrm{~cm}$ thick and $50.0 \mathrm{~cm}$ in diameter. The cylinders $\mathbf{A}$, with an outer radius $a(4.9 \mathrm{~cm})$, and $\mathbf{B}$, with an inner radius $b(15.3$ $\mathrm{cm}$ ), are made of copper, while the cylinder $\mathbf{C}$ with an inner radius of $22.8 \mathrm{~cm}$ is made of brass. The bottom disk $\mathbf{D}$ is fixed on a turntable $\mathbf{E}$ which rotates anticlockwise about a vertical axis. The rotation rate $\Omega$ of the turntable is variable from 1 to 20 r.p.m. and controlled with an accuracy of \pm 0.01 r.p.m..

The temperatures of the cylindrical walls $\mathbf{A}$ and $\mathbf{B}$ are kept constant by the running cold water which circulates through a constant temperature water bath WB. The cold water is kept at a constant temperature $T_{c}\left(15.00^{\circ} \mathrm{C}\right)$ within a limit of accuracy of $\pm 0.01^{\circ} \mathrm{C}$. A working fluid is contained in the annular space between the walls $\mathbf{A}$ and $\mathbf{B}$. In this experiment, aqueous

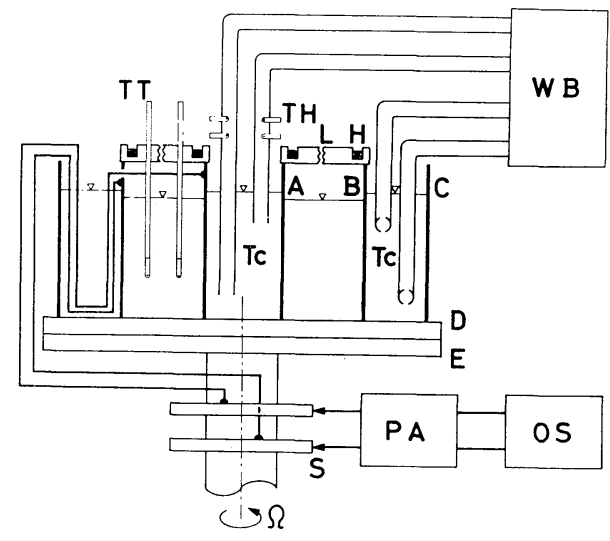

Fig. 1 Schematic diagram of experimental apparatus. Explanation is given in the text.

copper sulfate solution (the concentration of $\mathrm{CuSO}_{4}$ is about $0.02 \%$ ) is used as the working fluid. The depth $d$ is set at $15.0 \mathrm{~cm}$. A 2.00 $\mathrm{KHz}$ alternating voltage generated by the $\mathrm{RC}$ oscillator $\mathbf{O S}$ is applied across the cylinders $\mathbf{A}$ and $\mathbf{B}$ via the power amplifier $\mathbf{P A}$ and the slip rings $\mathbf{S}$ for the internal heating. To insulate the cylinders electrically from the cooling water, the inner surface of $\mathbf{A}$ and the outer surface of $\mathbf{B}$ are coated with lacquer with a thickness of about $0.1 \mathrm{~mm}$.

Temperature distributions in the fluid layer are measured by six thermistors TT. They are set along a radius of the lid $\mathbf{L}$, which is made of transparent acrylic resin with a thickness of 3.0 $\mathrm{cm}$. Since the lid is fixed on the convection chamber, the thermistors TT rotate with the turntable E. The electrical signals from the thermistors are fed out of the rotating system via mercury slip rings $\mathbf{H}$. Each thermistor sensor of $1.5 \mathrm{~mm}$ in diameter with a time constant of 0.2 sec in water is set at the tip of a stainless tube of $2.1 \mathrm{~mm}$ in diameter. A Wheatstone bridge with this thermistor as its arm is constructed so that the temperature distribution in the fluid layer is detected as the unbalanced voltage of the bridge. The voltage is recorded on a multi-pen recorder and converted into temperature. The temperature is read with a resolution of $0.01^{\circ} \mathrm{C}$.

The heat generated in the fluid layer causes a small temperature difference between the inlet and the outlet of the water which circulates through the inner cylinder $\mathbf{A}$. It is detected by thermistors TH which are similar to TT. The rate of heat transport to the inner wall is known from this temperature difference. When the 
measurements of heat transport are carried out or the photographs of top-surface flow patterns are taken, another lid without mercury slip rings is used instead of the lid $\mathbf{L}$.

\section{General characteristics of convective motions}

\subsection{Top-surface flow patterns}

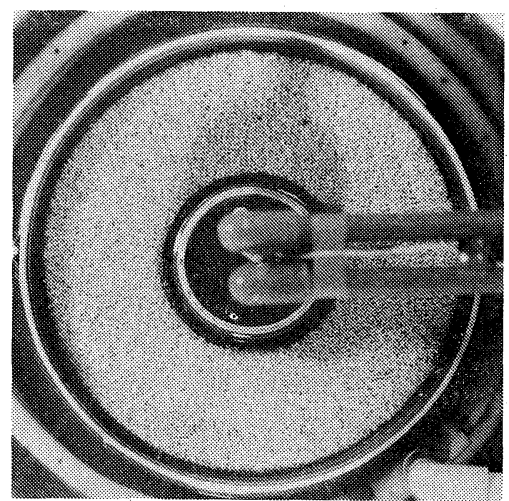

Fig. 2 (a)

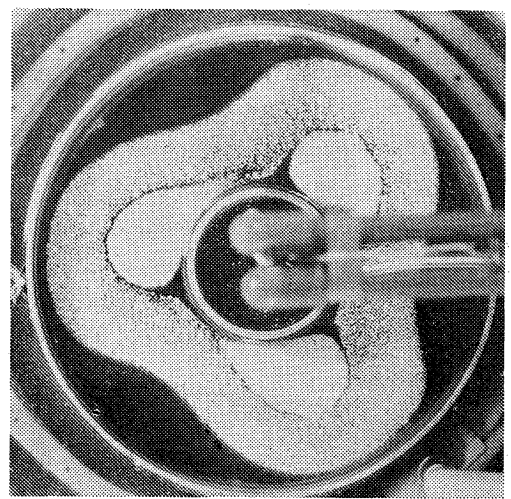

Fig. 2 (c)
Since the cylindrical walls of the convection chamber are made of copper with a high electric conductivity, the electrical power input $P$ may be identified to the rate of heat generation in the fluid layer. When the heating rate $P$ is kept constant, the flow patterns mainly depend on the rotation rate $\Omega$. The flow is axisymmetric zonal

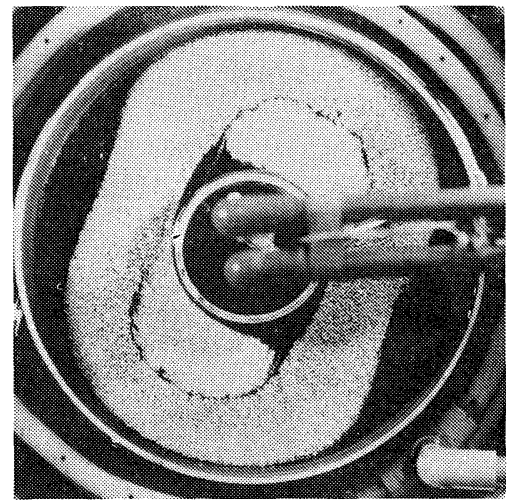

Fig. 2 (b)

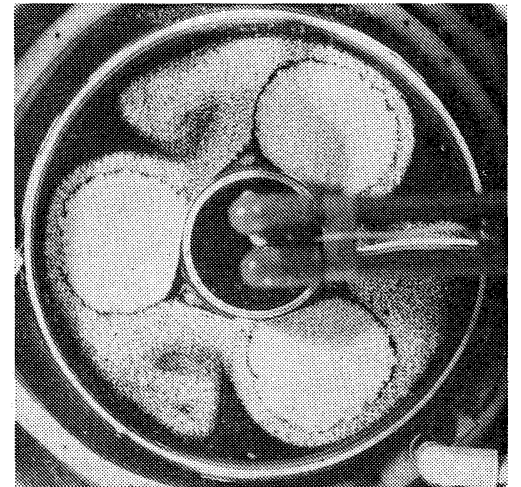

Fig. 2 (d)

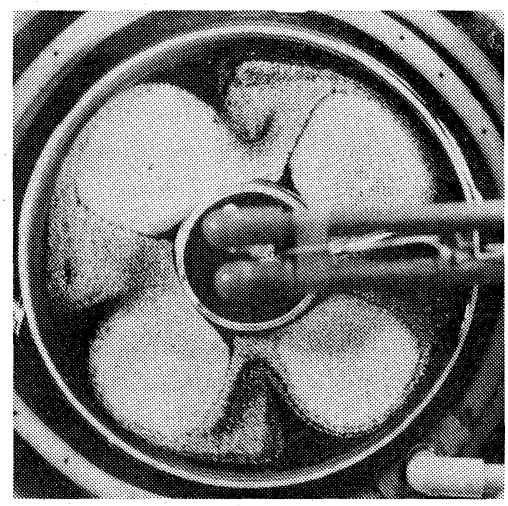

Fig. 2 (e)

Fig. 2 Examples of the top-surface flow patterns for $d=15.0 \mathrm{~cm}$ and $P=50.0$ watts. (a) Case 017, (b) Case 217, (c) Case 320, (d) Case 338 and (e) Case 445. Case 217, for example, means the flow of the wave number $m=2$ which takes place for rotation rate $\Omega=1.7$ r.p.m.. 
for small rotation rates, but it gives way to a wave motion at a certain critical rotation rate. The wave number $m$ tends to increase as $\Omega$ increases.

Examples of the top-surface flow are given in Figs. 2, where the heating rate $P$ is kept at 50.0 watts. In the following let us classify various flows obtained at $P=50.0$ watts by numbers of three figures giving values of $m$ and $\Omega$. For example, we designate a flow of $m=2$ which takes place at $\Omega=1.7$ r.p.m. by Case 217 . Plastic particles are used in order to visualize flow patterns. Fig. 2(a) shows the axisymmetric zonal flow, the azimuthal velocity of which is directed anticlockwise in the narrow region near the inner wall, but clockwise in the outer wide region (see Fig. 17), as observed by Hide and Mason (1970). On the other hand, Figs. 2(b) and (c) are cases of the wave motions. Plastic particles gather locally in clockwise rotating vortices which are surrounded by the meandering stream flowing clockwise (see Figs. 18). Figs. 2(d) and (e) show the cases for higher rotation rates, where not only clockwise vortices but also anticlockwise vortices grow intense and the meandering stream almost disappears.

\subsection{Transition curves}

Transition points between the axisymmetric zonal flow and the wave motion were measured for various values of $\boldsymbol{P}$ by varying $\Omega$ gradually. ${ }^{(1)}$ Near the transition point, the increment or decrement of $\Omega$ was less than 0.02 r.p.m. and the time interval of varying $\Omega$ was more than 40 minutes. The result is shown in Fig. 3. The broken line is the transition curve from the axisymmetric zonal flow to the wave motion, above which the axisymmetric zonal flow is unstable for some disturbances. On the other hand, the solid line is the transition curve from the wave motion to the axisymmetric zonal flow, below which the axisymmetric zonal flow is stable for any disturbances. The regime between the two transition curves is the metastable regime, where observed flow patterns depend on the initial states;

(1) When the measurements of the transition point (and also of the drift velocity of the vortex disturbances presented in the following Section 3.3) were carried out, use was made of only one of six thermistor thermometers whose sensor was set at the place $[R=6.0 \mathrm{~cm}$ and $z=13.5 \mathrm{~cm}$ (see Fig. 6)] where the amplitude of the temperature variation becomes maximum when wave motions develop.

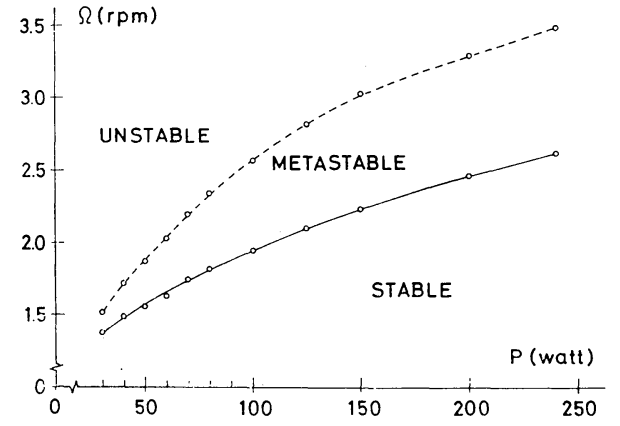

Fig. 3 Stability diagram. The ordinate is rotation rate $\Omega$ and the abscissa is the internal heating rate $P$. "Metastable" means the state in which either axisymmetric flow or wave motion takes place under the same external conditions.

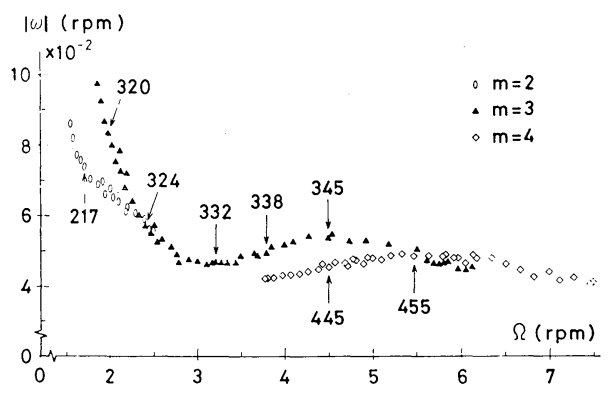

Fig. 4 Dependsnce of the angular velocity of the vortex drift $\omega$ on rotation rate $\Omega . \omega$ is measured relative to the container. $P=50.0$ watts. The numbers by the arrows in this figure denote the cases for which the structure analysis was carried out in this study. The arrows themselves indicate the corresponding positions on the $|\omega|-\Omega$ curves.

that is, either axisymmetric zonal flow or wave motion takes place under the same external conditions.

\subsection{Drift velocities of vortex disturbances}

The vortex disturbances, such as shown in Figs. 2 , drift in the clockwise direction relative to the convection chamber. The relationship between the drift angular velocity $\omega$ and the rotation rate $\Omega$ is shown in Fig. 4, where $P$ is kept at 50.0 watts. Since $\omega$ is always negative in this experiment, the ordinate indicates the absolute value of $\omega$. As is evident from this figure, the curve for $m=3$ has a minimum and a maximum, being not monotonic. Dependence of $\omega$ on $\Omega$ for $m=2$ and for $m=4$ is similar to that on smaller $\Omega$ and on larger $\Omega$ of the curve for $m=3$ respec- 
tively. The drift angular velocity is two orders smaller than the angular velocity of the basic rotation.

\subsection{Radial heat transport}

The rate of radial heat transport to the inner wall $Q_{\text {in }}$ was measured for various $\Omega$ under the condition for $P=50.0$ watts. The results are shown in Fig. 5. Each plot in the figure is the average of several measurements for the corresponding $\Omega . Q_{\text {in }}$ is roughly 20 watts (about $40 \%$ of the total input $P$ ) through the whole range of $\Omega$ measured. If examined in detail, however, $Q_{\text {in }}$ tends to increase monotonically with $\Omega$ for axisymmetric zonal flows, ${ }^{(2)}$ and has the minimum at $\Omega \fallingdotseq 3$ r.p.m. and the maximum at $\Omega \fallingdotseq 4.5$ r.p.m. for wave motions of $m=3$. It is worth while noting that this dependence of $Q_{\text {in }}$ on $\Omega$ in the range of wave motion is very similar to the dependence of $|\omega|$ on $\Omega$.

\section{Thermal structure}

4.1 Method of analysis of temperature fields The temperature distributions in the fluid layer were measured by above-mentioned six thermistors, which could be moved stepwise either upward or downward. The locations of the sensors

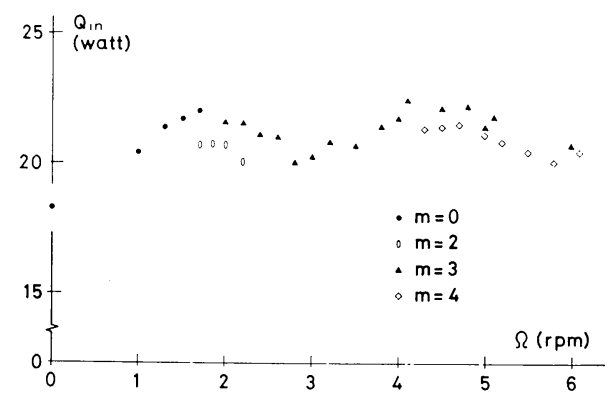

Fig. 5 Dependence of the rate of heat transport to the inner wall $Q_{\text {in }}$ on rotation rate $\Omega . P=50.0$ watts.

(2) This tendency is compatible with the result obtained by Hide and Mason (1970). They expressed the rate of radial heat transport with the Nusselt number $N$ defined as

$$
N=\frac{P \ln (b / a)}{16 \pi k d\left(T_{m}-T_{c}\right)}
$$

where $k$ is the thermal conductivity of the fluid and $T_{m}$ is the mean temperature on the cylindrical wall at $R=\sqrt{a b}-a \fallingdotseq(b-a) / 2$. As will be shown later, $T_{m}$ rises monotonically with $\Omega$ for axisymmetric zonal flows so that $N$ decreases in agreement with their result.

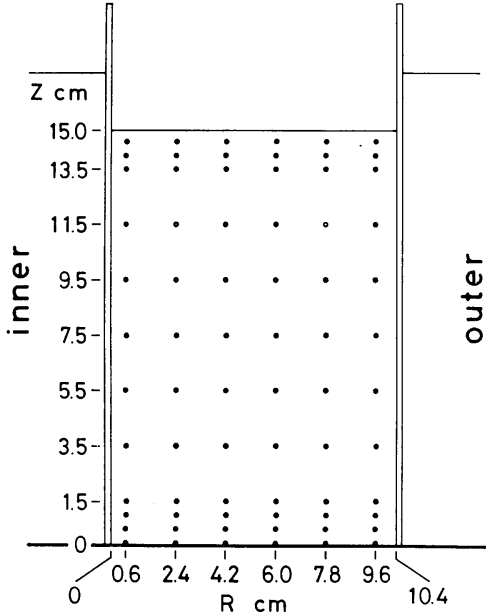

Fig. 6 Locations of the measuring points of the temperature. $R$ : the distance from the inner wall; $z$ : the distance from the bottom.

are shown by black points in Fig. 6. When the flow is axisymmetric, the temperature records are flat lines. But, when the flow is the steady wave motion, the records are regular wavy curves. This is due to the fact that the drifting vortices pass through the sensors one after another. The records for steady waves were read at intervals of $1 / 24$ of each wave length (at intervals of $15^{\circ}$ with respect to the phase angle).

Since the vertical distance between the measuring points was $2.0 \mathrm{~cm}$ in the middle layer, the intermediate temperature was obtained with a proportional allotment method. Let us denote the temperature by $T(R, \varphi, z)$, where $R$ is the distance from the inner wall, $z$ is the height and $\varphi$ is the phase angle. We assume that it may be expressed with cosine series as follows:

$$
T(R, \varphi, z)=\sum_{n=0}^{5} A_{n}(\varphi, z) \cos \frac{n \pi}{b-a} R,
$$

where $A_{n}(n=0 \sim 5)$ are coefficients to be determined with the data obtained by thermistor thermometers. This expression implies that the radial temperature gradients vanish at the inner and outer walls so that there are no heat transport across the walls. Nevertheless, by the use of this expression, the temperature is smoothly interpolated throughout the fluid layer, whereas the temperature distributions in the side-boundary layers are not dealt with in this analysis. After these interpolations, the temperature $T(R, \varphi, z)$ is divided into two parts: the zonal mean tempera- 
ture and the deviation from it.

\subsection{Temperature distributions in the meridio- nal plane}

The temperature distributions in the meridional plane for axisymmetric flows are shown in Figs. 7. Fig. 7(a) shows the distribution for Case 000, which is the special case of zero rotation rate. This figure indicates that the isotherms are almost horizontal except for the bottom region and that the vertical temperature gradient is slightly larger in the upper layer than in the lower layer. Fig. 7 (b) shows the distributions for axisymmetric zonal flows. We see from this figure that with the increase of the rotation rate, the slopes of the isotherms increase and the temperature gradients become larger not only in the horizontal but also in the vertical directions.

Figs. 8(a) and (b) show the distributions of the zonal mean temperature for wave motions of $m=$ 3 obtained for various values of $\Omega$. As the rotation rate increases, the temperature at the uppermost layer rises and the vertical temperature gradient becomes larger. It is also seen that, with the increase of the rotation rate, the middle parts of the isotherms are depressed gradually, i.e., the region nearby the mean radius tends to become warmer than the inner and outer regions at the same height. This temperature profile is attributed to the effect of internal heating and very different from the profile obtained in the wall-heated system (Matsuwo et al., 1976), in which the isotherms are almost linear.

\subsection{Eady's criterion}

As already mentioned, in the metastable regime, either axisymmetric zonal flow or wave motion

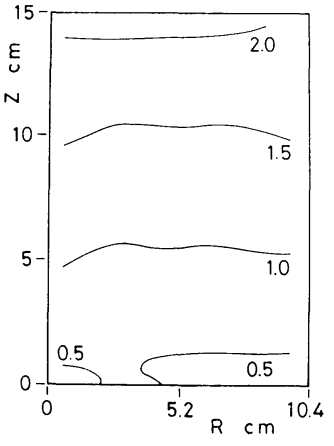

(a)

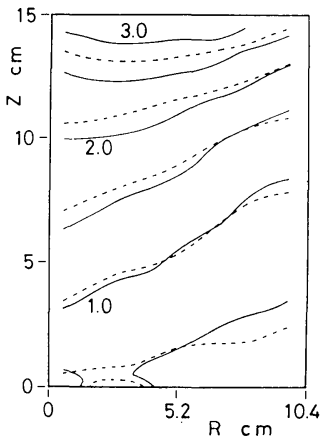

(b)
Fig. 7 Temperature distributions (represented by the deviation from $T_{c}=15.0^{\circ} \mathrm{C}$ ) for axisymmetric flows. The unit is ${ }^{\circ} \mathrm{C}$. (a) Case 000, (b) Case 013 (broken lines) and Case 017 (solid lines).

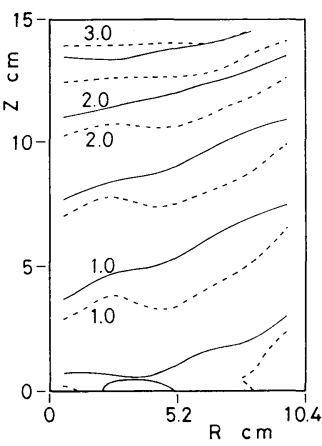

(a)

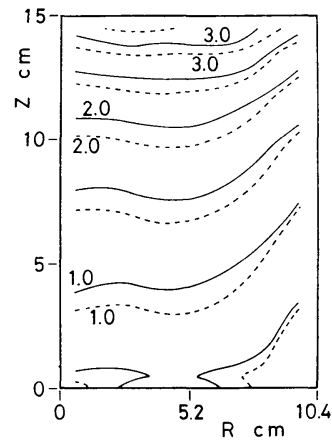

(b)
Fig. 8 Distributions of the zonal mean temperature (deviation from $T_{c}=15.0^{\circ} \mathrm{C}$ ) for wave motions. The unit is ${ }^{\circ} \mathrm{C}$. (a) Case 320 (solid lines) and Case 332 (broken lines), (b) Case 338 (solid lines) and Case 345 (broken lines).

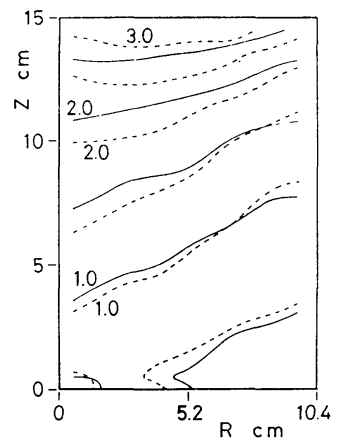

Fig. 9 Distributions of the temperature (deviation from $T_{c}=15.0^{\circ} \mathrm{C}$ ) for Case 017 (broken lines) and the zonal mean temperature for Case 217 (solid lines). The unit is ${ }^{\circ} \mathrm{C}$.

takes place under the same external conditions. For example, at a rotation rate of $\Omega=1.7$ r.p.m., Case 017 (axisymmetric zonal flow) and Case 217 (wave motion) are observed. Fig. 9 shows the meridional distribution of the temperature for Case 017 and that of zonal mean temperature for Case 217. According to Eady's criterion (Eady, 1949; Fowlis and Hide, 1965; Hide, 1970), when the value of $B$ defined as

$$
B=\frac{g \alpha\left(\partial T_{0} / \partial z\right) d^{2}}{4 \Omega^{2}(b-a)^{2}}
$$

becomes less than 0.58 , the axisymmetric zonal flow gives way to the wave motion, where $g$ is the acceleration of gravity; $\alpha$ is the coefficient of thermal expansion; $\partial T_{0} / \partial z$ is the uniform vertical temperature gradient of the basic state. The value of $B$ for Case 017 and Case 217 (say, $B_{017}$ and $B_{217}$ respectively) were determined as $B_{017}=$ 0.63 and $B_{217}=0.53$ by the use of the mean 
vertical temperature gradient in the main domain $(2.4 \mathrm{~cm} \leqq R \leqq 7.8 \mathrm{~cm}, \quad 1.5 \mathrm{~cm} \leqq z \leqq 13.5 \mathrm{~cm}) \quad$ as $\partial T_{0} / \partial z$ (see Table 1). Hence, as $B_{017}>0.58>B_{217}$, Case 017 and Case 217 satisfy Eady's criterion for baroclinic instability.

The thermal structure of the vortex disturbances for Case 217 is shown in Figs. 10 and 11. Fig. 10 shows the vertical cross section of the temperature deviation from the zonal mean along the circle of $R=6.0 \mathrm{~cm}$. Horizontal distributions of the temperature deviation are shown for two different levels in Figs. 11. We see from these figures that the maximum amplitude is located in the uppermost layer and the axes incline forward (in the direction of the vortex drift) with height. These features agree well with characteristics of the baroclinic wave which were indicated by Eady (1949) and confirmed by the wallheated experiment (Matsuwo et al., 1976). Therefore, judging from the mean temperature field, the direction of the vortex drift, and the inclination of the axes, we can conclude that these vortices are of the same kind as the disturbances developing in the wall-heated system with the inner-wall heated.

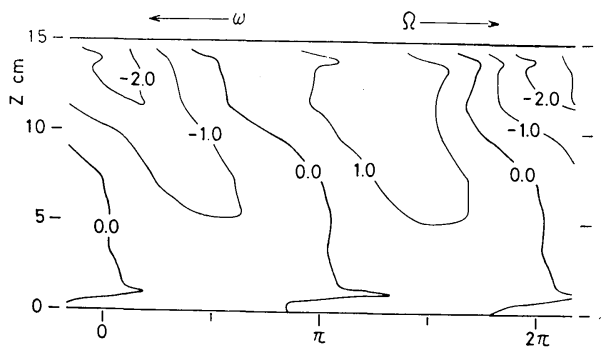

Fig. 10 Vertical cross section of the temperature deviation along the circle of $R=$ $6.0 \mathrm{~cm}$ for Case 217. The unit is $10^{-1}{ }^{\circ} \mathrm{C}$.

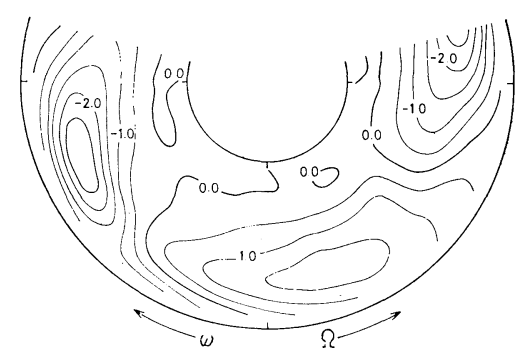

(a)

\subsection{Dependence of the vortex structure on the rotation rate}

Figs. 12 15 show the thermal structures for Cases 324, 332 and 345 . The wave number in these three cases is 3 in spite of the different rotation rates. Figs. 12 show the vertical cross sections of the temperature deviations along the circle of $R=6.0 \mathrm{~cm}$, while the horizontal distributions at two different levels are presented in Figs. 13 and 14. The distributions of the amplitude are shown in Figs. 15. These figures indicate that the thermal structure of the vortex disturbance depends on the rotation rate as follows.

Table 1. Vertical stability and phase difference.

\begin{tabular}{lccc} 
Case & $\Delta T_{0}\left({ }^{\circ} \mathrm{C}\right)$ & $B$ & $\Delta \varphi$ (degree) \\
000 & 1.32 & - & - \\
013 & 1.99 & 0.96 & - \\
015 & 2.12 & 0.78 & - \\
017 & 2.21 & 0.63 & - \\
217 & 1.88 & 0.53 & -38 \\
220 & 1.81 & 0.37 & -30 \\
320 & 1.83 & 0.38 & -68 \\
324 & 1.81 & 0.26 & -45 \\
332 & 1.98 & 0.16 & -30 \\
338 & 2.16 & 0.12 & -38 \\
345 & 2.21 & 0.090 & -38 \\
445 & 2.37 & 0.096 & -38 \\
455 & 2.45 & 0.067 & -30 \\
\hline
\end{tabular}

$\Delta T_{0}$ : mean vertical temperature difference between two levels at $z=13.5 \mathrm{~cm}$ and $z=$ $1.5 \mathrm{~cm}$ in the main domain $(2.4 \mathrm{~cm} \leqq R \leqq$ $7.8 \mathrm{~cm})$.

B: dimensionless measure of the vertical stability $\left(=\frac{g \alpha\left(\partial T_{0} / \partial z\right) d^{2}}{4 \Omega^{2}(b-a)^{2}}\right)$.

$\Delta \varphi:$ phase difference of the cold axis between two levels at $z=11.5 \mathrm{~cm}$ and $z=5.5 \mathrm{~cm}$.

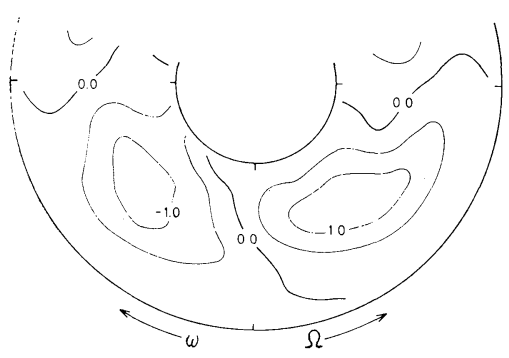

(b)

Fig. 11 Distributions of the temperature deviation on horizontal planes at (a) $z=11.5 \mathrm{~cm}$ and (b) $z=5.5 \mathrm{~cm}$ for Case 217. The unit is $10^{-1}{ }^{\circ} \mathrm{C}$. 


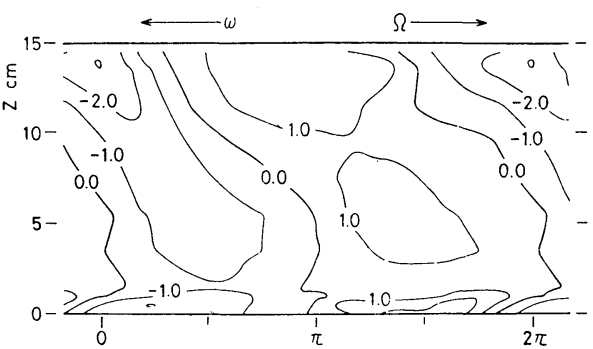

Fig. 12 (a)

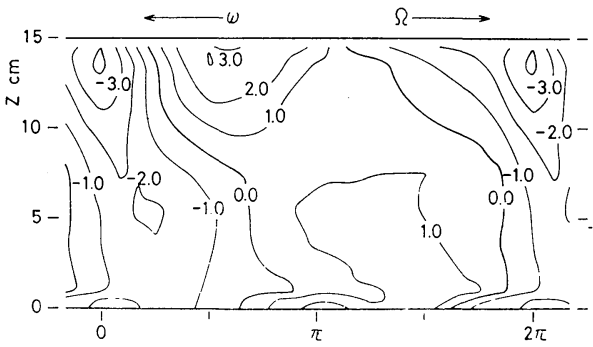

Fig. 12 (b)

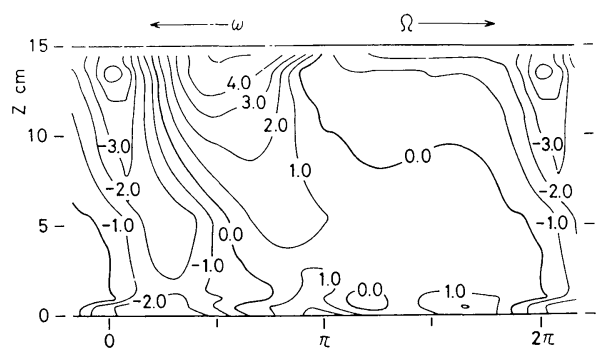

Fig. 12 (c)

Fig. 12 Vertical cross sections of the temperature deviation along the circle of $R=6.0 \mathrm{~cm}$. The unit is $10^{-1}{ }^{\circ} \mathrm{C}$. (a) Case 324 , (b) Case 332 and (c) Case 345.

1) The amplitude of the temperature deviation tends to increase as the rotation rate increases, in agreement with the result obtained by Matsuwo et al. (1976).

2) The axes of the temperature deviation incline outward with height at a low rotation rate. But, with the increase of the rotation rate, the inclinations decrease and cold and warm axes shift outward and inward respectively.

3) If we restrict our attention to the cold axis which is more distinguishable than the warm axis, we may say that the vortex disturbance with the minimum $|\omega|$ has the least inclined axis in the drift direction. Further, as to the disturbances with the same value of $|\omega|$, we may say that the disturbance for a higher $\Omega$ inclines less forward

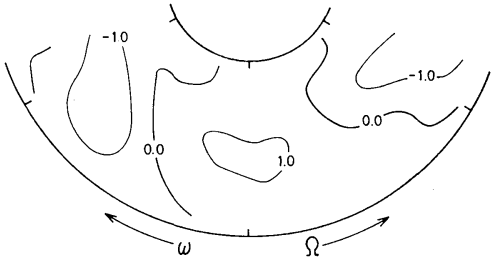

Fig. 13 (a)

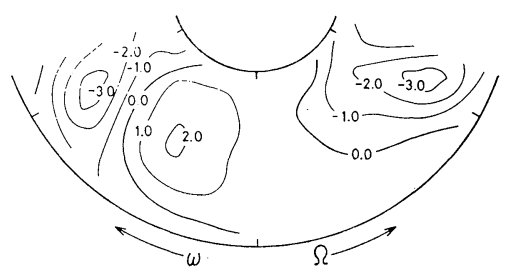

Fig. 13 (b)

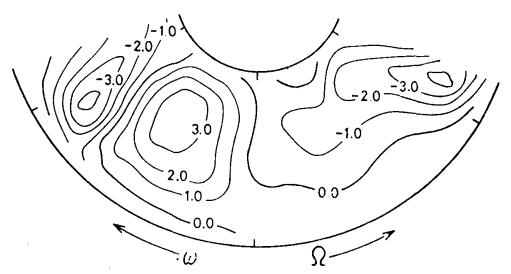

Fig. 13 (c)

Fig. 13 Distributions of the temperature deviation on the horizontal plane at $z=11.5 \mathrm{~cm}$. See Fig. 12 for additional explanations.

than that for a lower $\Omega$ (see Table 1 and Fig. 4).

4) At higher rotation rates, the secondary disturbance appears in each of the warm and the cold regions. The secondary disturbance in the warm region is situated in the lower layer (see Figs. 14(c) and 12(c)), while that in the cold region is situated in the upper layer (see Figs. 13(c) and 12(c)).

As to the cases of $m=3$, Case 320 and Case 338 are similar to Case 217 and Case 345 respectively. On the other hand, as to the cases of $m \neq 3$, Case 220 is similar to Case 324 , while Cases 445 and 455 are similar to Case 345. That is to say, the cases in the similar position on the $|\omega|-\Omega$ curves have the similar structure. This is consistent with the tendency obtained in the wallheating experiment (Matsuwo et al., 1976), namely, that the temperature distribution for a large wave number is more or less similar to that for a smaller wave number at a lower rotation rate.

\subsection{Disturbances near the bottom}

Near the bottom, both cold and warm regions separate into two or three parts. Such separations 
in the wall-heated system have been already pointed out in a numerical experiment (Tokioka, 1970) and confirmed by the laboratory experiment (Matsuwo et al., 1976, 1977). Thus, in the lower layer the isolines of the temperature deviation are so complicated that the shape and the

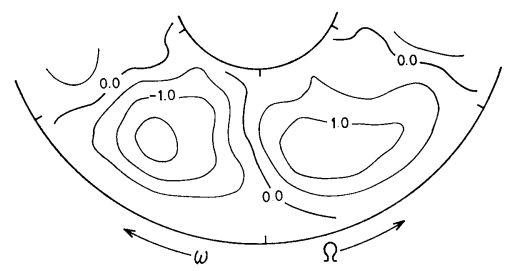

Fig. 14 (a)

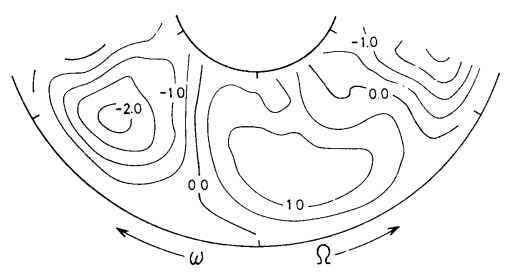

Fig. 14 (b)

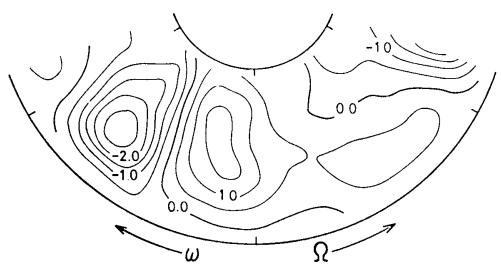

Fig. 14 (c)

Fig. 14 Same as Figs. 13, but for at $z=5.5 \mathrm{~cm}$. intensity of each region vary with height remarkably. In addition to these facts, the absolute value of the temperature deviation along the axis of the main disturbance in each region reaches its minimum nearby the height of $z=1.5 \mathrm{~cm}$ in the most cases. Accordingly, it is very difficult to follow the axes throughout the fluid layer.

Figs. 16 show the temperature deviations near the bottom for Case 332, which has rather little variations of the thermal structure with height. We see from Figs. 16(b) and (c) that the main axis of the cold region inclines backward (in the opposite direction of the vortex drift) with height. This is the modification of the vortex structure caused by forming the Ekman layer balance (Tokioka, 1970). There are three peaks in the warm region in Fig. 16(c). The outside peak is the lowest part of the main disturbance and the middle peak corresponds to the secondary disturbance which may be traced up to the height of $z=9.5 \mathrm{~cm}$. The axes of these two disturbances also incline backward with height. The inside peak is the fairly strong one corresponding to the disturbance with its maximum at nearby $z=1.0$ $\mathrm{cm}$, but it is too short to be discerned its existence at $z=3.5 \mathrm{~cm}$. Since its axis inclines inward and forward with height, one may identify this disturbance with the disturbance reported by Green (1960). But the height distribution of the mean zonal flow is not perceived as parabolic (see Fig. 19(b)), so that we can not say that Green's dynamic condition is satisfied.

\section{Dynamical structure}

\subsection{Method of determination of dynamical quantities}

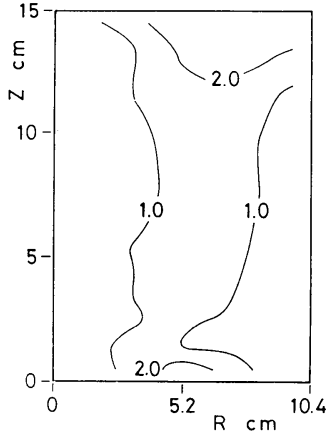

(a)

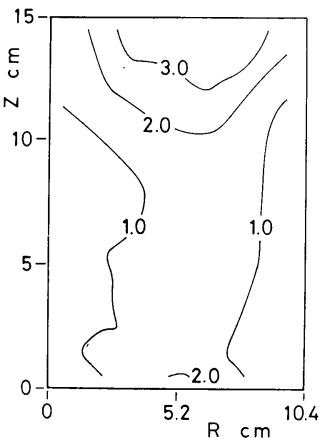

(b)

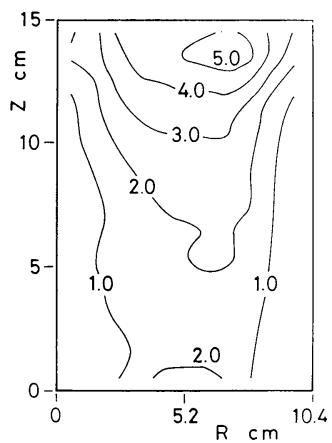

(c)

Fig. 15 Distributions of the temperature amplitude defined as $\left(T_{\max }^{\prime}-T_{\min }^{\prime}\right) / 2$, where $T_{\max }^{\prime}$ and $T_{\min }^{\prime}$ are the maximum and the minimum of the temperature deviation respectively. See Fig. 12 for additional explanations. 


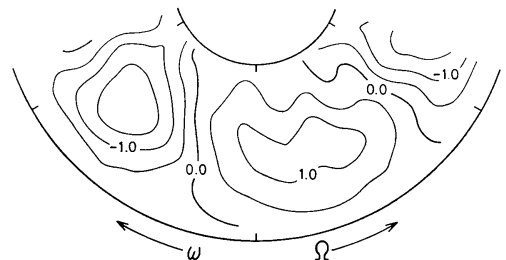

Fig. 16 (a)

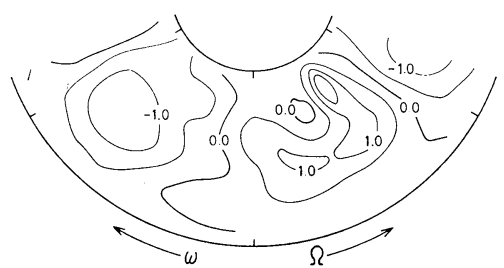

Fig. 16 (b)

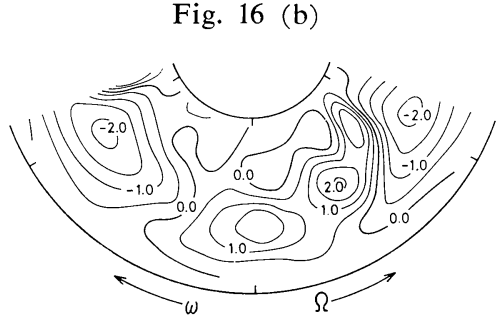

Fig. 16 (c)

Fig. 16 Distributions of the temperature deviation on horizontal planes at (a) $z=3.5 \mathrm{~cm}$, (b) $z=1.5 \mathrm{~cm}$ and (c) $z=0.5 \mathrm{~cm}$ for Case 332 . The unit is $10^{-1}{ }^{\circ} \mathrm{C}$.

By making use of the temperature distributions in the fluid layer and the velocity fields at the top-surface, we can describe the dynamical structure of the fluid layer, though it is based on a few assumptions stated below.

Although the working fluid used in this experiment is aqueous copper sulfate solution, its concentration is very dilute. Thus, we use the density $\rho$, the specific heat $c$ and the coefficient of thermal expansion $\alpha$ of water as those of the working fluid and neglect the diffusion coefficients such as thermal diffusivity and kinematic viscosity. The observed horizontal velocity associated with fully developed wave motions is $0.7 \mathrm{~cm} / \mathrm{s}$ at the most. Hence, the corresponding Rossby number is 0.2 even for the case for the lowest $\Omega$. Accordingly, we can make the quasi-geostrophic approximation in the present analysis of the dynamical structure. The density variation due to height difference is negligible as compared with that due to thermal expansion. Therefore, we can use the Boussinesq approximation in this analysis.

The calculations of dynamical quantities closely follow the methods by Matsuwo et al. (1977). That is, the mean zonal velocity component $\bar{V}$ and the perturbed radial and zonal velocity components $u^{\prime}$ and $v^{\prime}$ are determined from the thermal wind formula and the top-surface velocity field. The vertical velocity component $w$ is calculated from the equation of heat transfer in which the internal heating rate per unit volume $q$

$$
\left(=\frac{P}{[2 \pi d \ln (b / a)] r^{2}}\right)
$$

is taken into account :

$$
c \rho \frac{D T}{D t}=q
$$

that is

$$
w=\frac{\frac{q}{c \rho}-u \frac{\partial T}{\partial r}-(v-r \omega) \frac{\partial T}{r \partial \theta}}{\partial T / \partial z}
$$

where $(r, \theta, z)$ are cylindrical coordinates fixed to the rotating system. As will be shown later, the mean radial velocity component $|\bar{U}| \ll|\bar{V}|,\left|u^{\prime}\right|$, $\left|v^{\prime}\right|$, so that we can neglect $\bar{U}$ in $u\left(=\bar{U}+u^{\prime}\right)$ in the above equation. In order to obtain the distribution of $\bar{U}$, use is made of the continuity equation in a meridional plane. It should be noted here that, when $\partial T / \partial z$ is small, we can not get reasonable values of $w$. Therefore, we should confine ourselves to qualitative discussions as to the distributions of $w^{\prime}, \bar{W}$ and $\bar{U}$. The pressure distributions are determined from the geostrophic relation at the top-surface and the hydrostatic equation. Furthermore, the rates of radial and vertical heat transport are estimated from the distributions of temperature and velocity in the fluid layer.

\subsection{Velocity fields}

The distributions of the horizontal velocity at the top-surface were obtained from the photographs taken by the use of a stroboscope. Fig. 17 shows the distribution for axisymmetric zonal flows. The weak anticlockwise flow is confined in the narrow region near the inner wall and the strong clockwise flow occupies the outer wide region, as indicated by Hide and Mason (1970). A point of inflection seems to be in existence slightly inside of the mean radius. The speed of the flow tends to increase with the increase of the rotation rate. Fig. 18(a) shows the distribution for the wave motion for a lower rotation rate. The clockwise vortices are enclosed with the wide meandering stream. Fig. 18(b) shows the distribution for a higher rotation rate, where 
the meandering stream almost disappears, while the clockwise vortices grow intense and the anticlockwise vortices become to be perceived. Hide and Mason (1970) showed the schematic illustrations of the top-surface flow patterns of steady waves developing under the various impressed

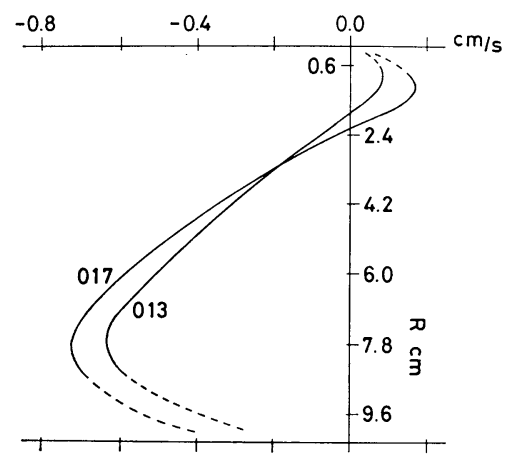

Fig. 17 Distributions of the top-surface azimuthal flow velocity for axisymmetric zonal flows, Case 013 and Case 017, based on photographs with a stroboscope.

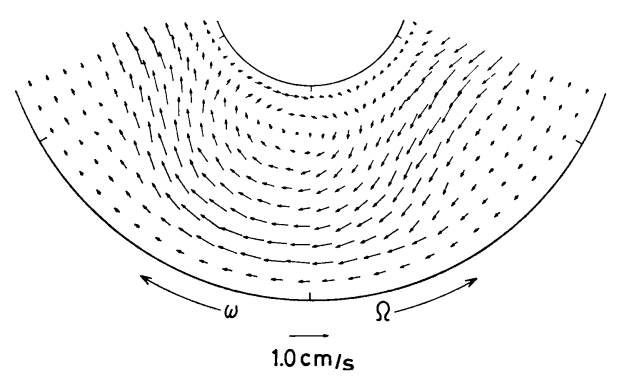

(a) temperature distributions in the radial direction. Compared with the illustrations obtained from their internal heating experiments, Fig. 18(b) corresponds to the case with both walls cooled expectedly, but Fig. 18(a) corresponds to the case with outer wall cooled. This is attributed to that the mean temperature distribution has the warmer region nearby the mean radius for a higher rotation rate, but nearby the inner wall for a lower rotation rate (see Figs. 8).

Figs. 19 are meridional cross sections of the mean zonal velocity of wave motions. The profile at the top-surface of Case 338 (also Case 332) is similar to that of the axisymmetric zonal flow, but the magnitude is considerably smaller than that of the axisymmetric zonal flow (see Fig. 17). Therefore, the mean zonal flow for $\Omega>\Omega_{m}$, as well as the axisymmetric zonal flows, has the distribution of the vertical component of vorticity with negative values over a wide region at the top-surface, which is due to the existence of the warmer region nearby the mean radius. This result is different from that obtained by Matsuwo

Fig. 18 Distributions of the top-surface horizontal flow velocity for wave motions. The length of arrows is proportional to the speed. The scale is shown in the figure. (a) Case 320 and (b) Case 338.

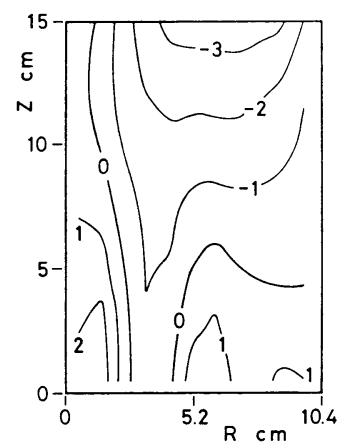

(a)

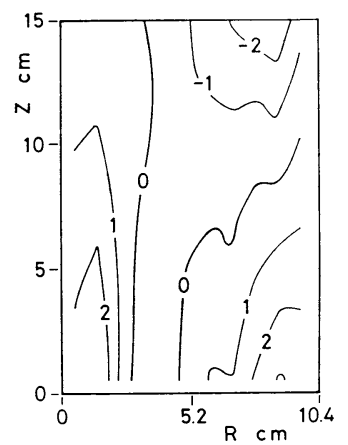

(b)

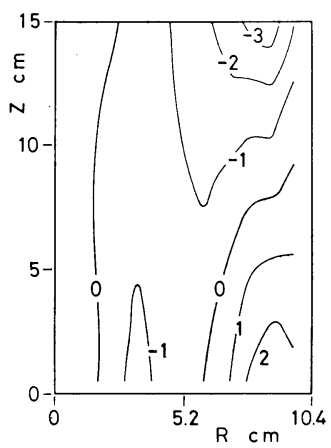

(c)

Fig. 19 Distributions of the mean zonal velocity. The unit is $10^{-1}$ $\mathrm{cm} / \mathrm{s}$. (a) Case 320, (b) Case 332 and (c) Case 338. 
et al. (1977) in the wall-heating experiment. They observed that the mean zonal flow at the topsurface is almost constant over a wide region except for the region very near the outer wall. Fig. 19(a) is rather similar to their result, although the flow direction is opposite to theirs. Figs. 19 show also that at the central region, the flow direction is clockwise and its value is nearly equal to the speed of the vortex drift. The vertical gradient of the velocity is negative almost throughout the entire region. The place where the magnitude of the gradient is maximum is located nearby the mean radius for Case 320 and it shifts outward with the increase of the rotation rate. The magnitude itself at that place increases as the rotation rate increases. As to Case 332, one may suppose that the mean zonal velocity has a parabolic height distribution near the inner wall, which is the dynamic condition for the disturbance reported by Green (1960). However, we can not discern such a distribution in Fig. 19(b).

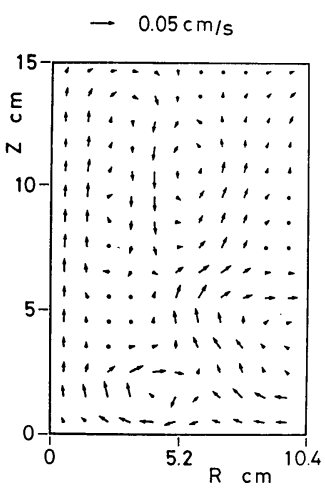

(a)

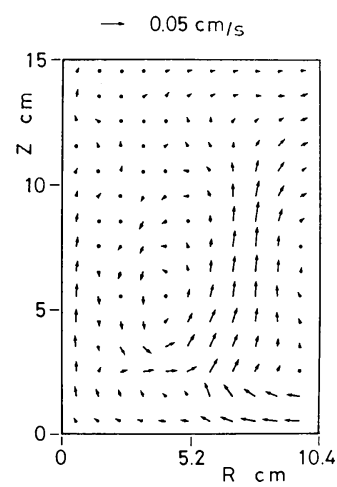

(b)

Fig. 20 Mean meridional flows. See Fig. 18 for additional explanations.

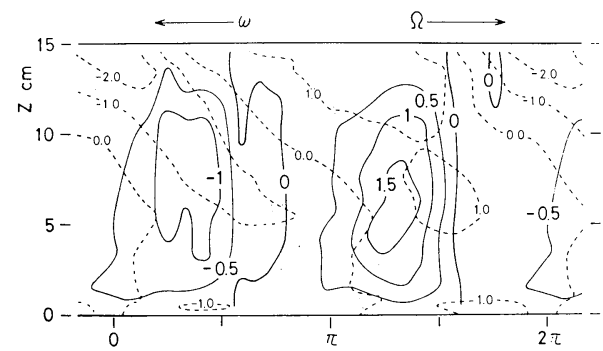

(a)
Figs. 20 show the mean meridional flows for wave motions. Although the flows are not simple, we can see that a small anticlockwise circulation is situated in the upper layer for Case 320 and the circulation grows to occupy the central region for Case 338.

The height distributions of vertical motion and temperature deviations along the circle of $R=$ $6.0 \mathrm{~cm}$ are shown in Figs. 21. Figs. 21(a) indicates that there are a descending and an ascending flows just in front of the cold and the warm axes respectively with each maximum around the middle height. This result is qualitatively in agreement with the result obtained in the wall-heating experiment (Matsuwo et al., 1977). The similar arrangements of vertical velocity and temperature deviations are seen also in Fig. 21(b), though the shape of each deviation is somewhat complicated. From such arrangements, we can expect the upward heat transport by the vortex disturbances.

\subsection{Pressure fields}

Fig. 22(a) shows the distributions of the pressure deviation at $z=15.0 \mathrm{~cm}$ and the temperature deviation at $z=14.5 \mathrm{~cm}$ of Case 338 . The pressure deviation corresponds to the surface displacement due to vortex motions. The magnitude of the displacement is $10^{-2} \mathrm{~mm}$ at the most. Since the fluid motion is regarded as geostrophic, positive and negative regions of the pressure deviation correspond to clockwise and anticlockwise vortices respectively. The temperature field at the top-surface is similar to that at $z=14.5 \mathrm{~cm}$. Therefore, we may conclude that the positive (or negative) regions of pressure and temperature deviations almost coincide with each other at the top-surface. Figs. 22(b) and (c) show the distributions of pressure and temperature deviations at the middle and the lower levels respectively. As

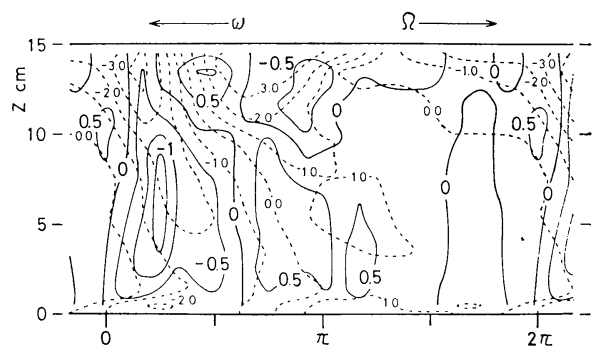

(b)

Fig. 21 Vertical cross sections of deviations of the vertical velocity (solid lines) and the temperature (broken lines) along the circle of $R=6.0 \mathrm{~cm}$. The units are $10^{-1} \mathrm{~cm} / \mathrm{s}$ for velocity and $10^{-1}{ }^{\circ} \mathrm{C}$ for temperature. (a) Case 320 and (b) Case 338. 
compared with the distributions of the temperature deviation, the distributions of the pressure

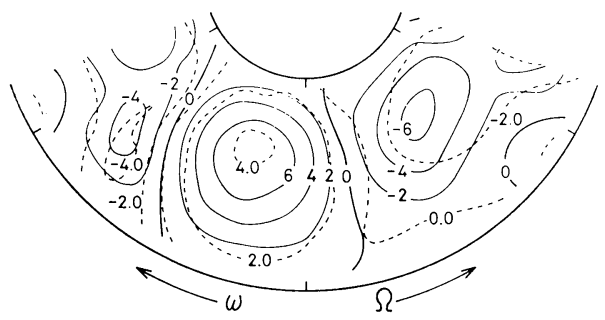

Fig. 22 (a)

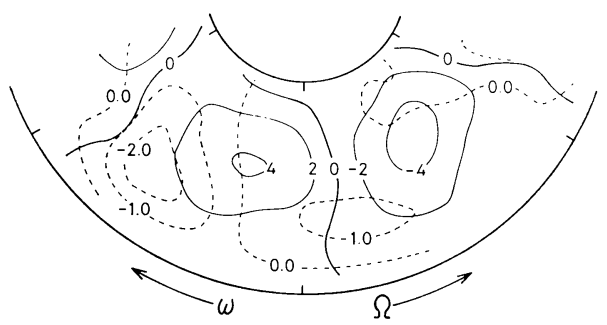

Fig. 22 (b)

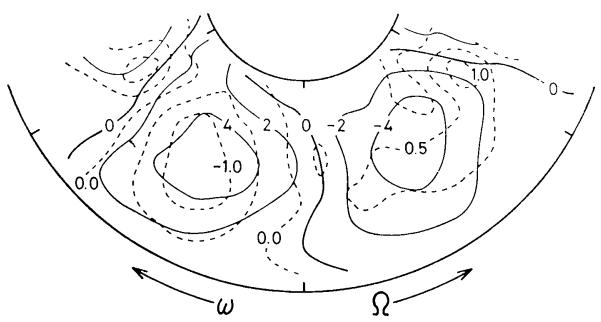

Fig. 22 (c)

Fig. 22 Distributions of deviations of the pressure (solid lines) and the temperature (broken lines) on horizontal planes at (a) $z=15.0$ $\mathrm{cm}$, (b) $z=7.5 \mathrm{~cm}$ and (c) $z=1.5 \mathrm{~cm}$ for Case 338. The temperature deviation at $z=14.5 \mathrm{~cm}$ is shown in (a) instead of that at $z=15.0 \mathrm{~cm}$. The units are $10^{-1} \mathrm{dyn} / \mathrm{cm}^{2}$ for pressure and $10^{-1}{ }^{\circ} \mathrm{C}$ for temperature.

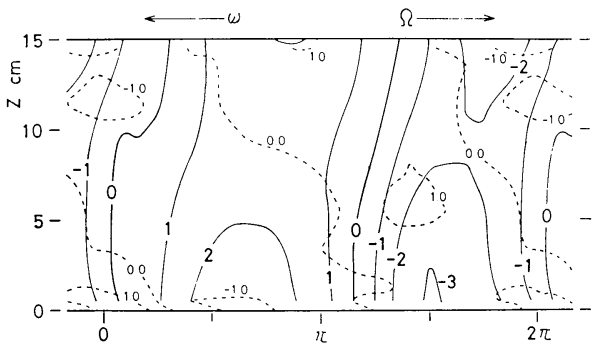

(a) deviation are fairly simple and vary little with height. Contrary to the distributions at the topsurface, at the lower layer, the positive region of the pressure deviation almost coincides with the negative region of the temperature deviation, and vice versa.

Figs. 23 present the vertical distributions of pressure and temperature deviations along the circle of $R=4.2 \mathrm{~cm}$. We see from these figures that the axes of the pressure deviation incline slightly backward with height in contrast with the axes of the temperature deviation. As to the trough line, its inclination tends to become smaller with the increase of the rotation rate, though the dependence of the inclination on the drift velocity is not discernible.

The meridional distributions of the amplitude of pressure deviation are shown in Figs. 24. The amplitude, with its minimum around the middle layer, increases with the rotation rate. The maximum is located in the lowermost layer for Case 320 (also Case 324) for a lower rotation rate, while in the uppermost layer for Case 338 (also Cases 332 and 345) for a higher rotation rate. The axes of the pressure deviation incline slightly inward with height in the radial direction and tend to shift inward as the rotation rate increases.

Matsuwo et al. (1977) analyzed the pressure field in the wall-heated system. However, unfortunately, their analysis was restricted to a case so that the dependence of the pressure field on the rotation rate was not presented. Nevertheless, the principal characteristics of the pressure fields described above agree with those obtained by them.

\subsection{Heat transport by vortex disturbances}

Figs. 25 present the rate of radial heat transport per unit vertical length by the vortex disturbances. The rate is positive in the main body and the location of the maximum rate

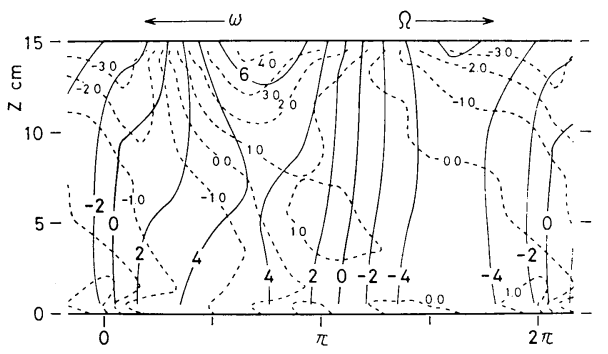

(b)

Fig. 23 Vertical cross sections of deviations of the pressure (solid lines) and the temperature (broken lines) along the circle of $R=4.2 \mathrm{~cm}$. The units are the same as those in Fig. 22. (a) Case 320 and (b) Case 338. 


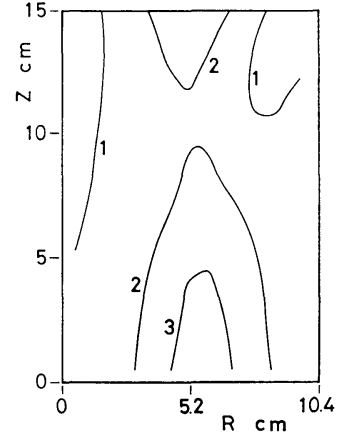

(a)

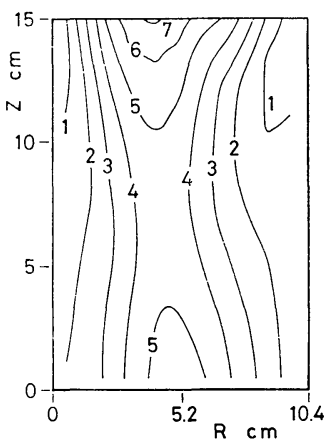

(b)
Fig. 24 Distributions of the pressure amplitude defined as $\left(p_{\text {max }}^{\prime}-p_{\text {min }}^{\prime}\right) / 2$, where $p_{\text {max }}^{\prime}$ and $p_{\text {min }}^{\prime}$ are the maximum and the minimum of the pressure deviation respectively. The unit is $10^{-1} \mathrm{dyn} / \mathrm{cm}^{2}$. (a) Case 320 and (b) Case 338 .

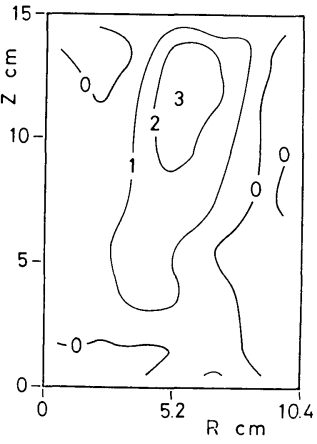

(a)

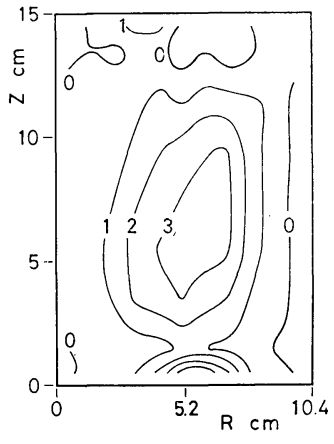

(b)
Fig. 25 Distributions of the rate of outward heat transport per unit vertical length by vortex disturbances. The unit is $\mathrm{J} / \mathrm{s} \cdot \mathrm{cm}$. (a) Case 320 and (b) Case 338.

nearly coincides with the region of the anticlockwise circulation in the meridional flow (see Figs. 20). Such a coincidence is seen in the wall-heated system (Matsuwo et al., 1977).

The rate of radial heat transport through cylindrical walls is shown in Fig. 26. Heat is transported outward and the maximum heat transport takes place slightly outside of the mean radius. It is worth to note that the most active outward heat transport is done by Case 332, because the measurement indicates that the minimum inward heat transport occurres at $\Omega \fallingdotseq 3$ r.p.m. (see Fig. 5), though this measurement includes the rate of heat transport by the mean meridional flow.

Fig. 27 shows the rate of vertical heat trans-

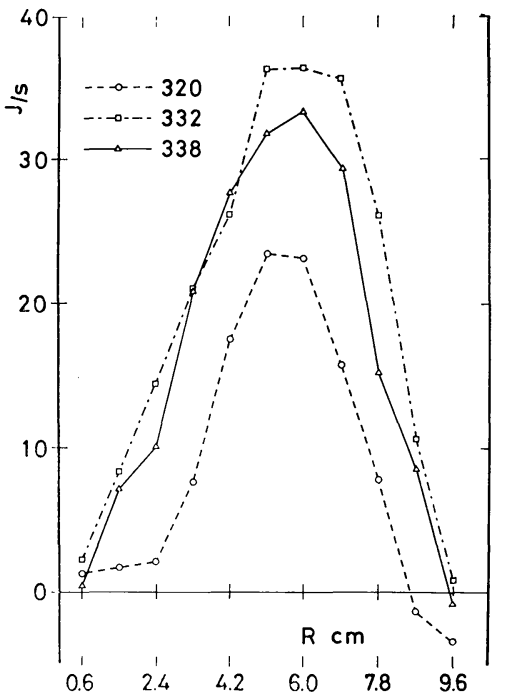

Fig. 26 Radial distributions of the rate of outward heat transport through cylindrical walls by vortex disturbances.

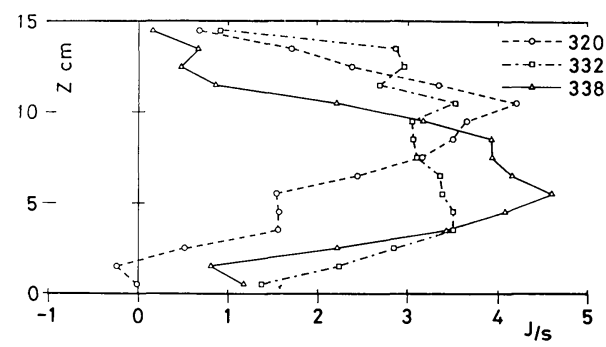

Fig. 27 Height distributions of the rate of upward heat transport through horizontal planes by vortex disturbances.

port through horizontal planes. As expected in Section 5.2, heat is transported upward. The maximum heat transport takes place in the upper layer for Case 320, while in the lower layer for Case 338. Each height of these layers also nearly coincides with the height of the center of the anticlockwise circulation in the corresponding mean meridional flow.

\section{Summary}

Convective motions in a rotating fluid annulus subject to internal heating and heat removal through both side-walls are studied experimentally. The general characteristics and the three-dimensional structure obtained are summarized as follows.

1) The measurements of the rate of radial heat transport to the inner wall and of the angular 
velocity of the vortex drift show that they depend on the rotation rate $\Omega$ and their dependence on $\Omega$ resembles each other. Each of them reaches its minimum at much the same rotation rate, say, $\Omega_{m}$.

2) When $\Omega<\Omega_{m}$, the distributions of the zonal mean temperature are almost linear in the radial direction with resemblance to those observed in the wall-heating experiments with the inner-wall heated. However, when $\Omega>\Omega_{m}$, the region nearby the mean radius becomes warmer than the inner and outer regions at the same height.

3) On the top-surface of the axisymmetric zonal flow, we see the weak anticlockwise flow confined in the narrow region near the inner wall and the strong clockwise flow occupying the outer wide region. Further, it seems that a point of inflection exists slightly inside of the mean radius.

4) In the wave motion for $\Omega<\Omega_{m}$, we observe the wide meandering stream associated with clockwise vortices. The stream, however, almost disappears for $\Omega>\Omega_{m}$, while the clockwise vortices grow intense and the anticlockwise vortices become to be perceived.

5) The meridional distribution of the mean zonal velocity indicates that, at the central region, the direction of the flow is clockwise and its value is nearly equal to the speed of the vortex drift. The mean zonal flow of the wave motion for $\Omega>\Omega_{m}$, as well as the axisymmetric zonal flows, has the distribution of the vertical component of vorticity with negative values over a wide region at the top-surface, which is due to the existence of the warmer region nearby the mean radius.

6) The amplitude of the temperature deviation, with its maximum in the uppermost layer, increases as the rotation rate increases. The axis inclines in the direction of the vortex drift with height and the inclination seems to reach its minimum at $\Omega_{m}$.

7) The amplitude of the pressure deviation, with its minimum around the middle layer, increases with the rotation rate. The maximum is situated in the lowermost layer for $\Omega<\Omega_{m}$, while in the uppermost layer for $\Omega>\Omega_{\mathrm{m}}$. The axis inclines in the opposite direction of the vortex drift with height and the inclination becomes smaller with the rotation rate. For each vortex disturbance, the inclination of the pressure deviation is smaller than that of the temperature deviation.

8) The positive (or negative) regions of pressure and temperature deviations almost coincide with each other at the top-surface. At the bottom, however, the positive region of the pressure deviation almost coincides with the negative region of the temperature deviation, and vice versa.

9) The vortex disturbances transport heat upward in the vertical direction and outward in the radial direction. The outward heat transport by the vortices seems to be the most active at $\Omega_{m}$. The position is slightly outside of the mean radius.

From these results, we can conclude that the fundamental structures of the vortex disturbances developing in the internally heated system are the same as those of the baroclinic waves analyzed by Matsuwo et al. $(1976,1977)$ in the wallheated system. Therefore, the present experiments support the indication by Hide and Mason (1970), namely, that the principal characteristics of the flow (in a rotating fluid annulus) are fairly insensitive to the radial dependence of heating and cooling, from the viewpoint of structure analysis.

\section{Acknowledgements}

The author would like to express his sincere thanks to Prof. R. Sawada and Prof. M. Uryu, Kyushu University, for their valuable comments and continuing encouragements throughout this work, and for their critical readings of the manuscript. The author is also indebted to Dr. N. Matsuwo, Kyushu University, for his helpful advices and encouragements. Thanks are extended to Mr. K. Tamaki for his assistance in experiments and drawing of all figures. Finally, the author must record his indebtedness to referees for their valuable comments and suggestions to improve the manuscript in many aspects.

This work was supported in part by Funds for Scientific Research from the Ministry of Education. The numerical computations were made by the use of FACOM 230-60 computer at Kyushu University.

\section{References}

Bowden, M. and H.F. Eden, 1965: Thermal convection in a rotating fluid annulus: Temperature, heat flow and flow field observations in the upper symmetric regime. J. Atmos. Sci., 22, 185-195.

Eady, E. T., 1949: Long waves and cyclone waves. Tellus, 1, 33-52.

Fowlis, W. W. and R. Hide, 1965: Thermal convection in a rotating annulus of liquid: Effect of viscosity on the transition between axisym- 
metric and non-axisymmetric flow regimes. J. Ketchum, C. B., 1972: An experimental study of Atmos. Sci., 22, 541-558.

Green, J.S. A., 1960: A problem in baroclinic stability. Quart. J. Roy. Meteor. Soc., 86, 237251.

Hide, R., 1958: An experimental study of thermal convection in a rotating liquid. Phil. Trans. Roy. Soc. Lond., A 250, 441-478. baroclinic annulus waves at large Taylor number. J. Atmos. Sci., 29, 665-679.

Matsuwo, N., M. Uryu and R. Sawada, 1976: An experimental study on the internal structures of baroclinic waves in a rotating annulus: Part I. Thermal structure. J. Meteor. Soc. Japan, 54, 339-350.

, 1970: In The global circulation of the atmosphere (ed. G. A. Corby). Roy. Meteor. Soc., London, pp. 196-221.

Hide, R. and P. J. Mason, 1970: Baroclinic waves in a rotating fluid subject to internal heating. Phil. Trans. Roy. Soc. Lond., A 268, 201-232.

Kaiser, J. A. C., 1969: Rotating deep annulus convection. Part 1. Thermal properties of the upper symmetric regime. Tellus, 21, 789-805.

1971: Heat transfer by symmetrical rotating annulus convection. J. Atmos. Sci., 28, 929932.

\title{
内部加熱によって回転流体中にひきおこされる 対流運動の熱的・力学的構造
}

\author{
宇 加 治 一 雄 \\ 福岡大学理学部応用物理学教室
}

半径方向に電流を流すことにより，内部的に加熱した回転流体の運動を実験的に調べ，流れの熱的・力学的構造を 明らかにした。

内部加熱に特有の結果として以下のことが指摘される。

（1）基本場の回転数 $\Omega$ に対して，内壁への熱輸送量と消擾乱のドリフトの速さは互いによく似た依存性を示し， どちらもほぼ同じ回転数 $\Omega_{m}$ で極小值に達する。

(2) $\Omega$ が $\Omega_{m}$ より大きくなると，方位角方向に平均した温度は，内外壁付近よりも同じ高さの平均半径付近の方 が高くなる。

（3）軸対称な帯状流及び $\Omega_{m}$ より大きな $\Omega$ で現れる波動の平均帯状流は，流体層の表面の広い範囲にわたって漓 度の負の鉛直成分をむつ。

(4) 加熱量一定の条件下で, 温度及び圧力擾乱の振幅は $\Omega$ とともに増大するが，擾乱による外向きの熱輸送量は $\Omega_{m}$ の近くで最も活発になり，またこのとき，温度擾乱の軸は最も鉛直に近ずく。

構造解析の結果, 内部的には加熱された回転流体に生ずる渦擾乱は Matsuwo et al. (1976，1977) によって解析 された外壁を加熱された回転流体中に生ずる傾圧不安定波と基本的に同じ構造をもつことが明らかになった。したが って，半径方向に温度勾配をもつ回転流体の主要な性質は冷・熱源の半径方向の配置の仕方にあまり影響されないと い5 Hide and Mason (1970) の指摘は, 構造解析の立場から支持される。 